\title{
A Reliability Model for a Two Dissimilar Units Series System with Repair Time-Dependent Standby
}

\author{
Jai Bhagwan, Amit Manocha, Anil Taneja
}

\begin{abstract}
The present paper stochastically analyze a system comprising two dissimilar units (unit-1/unit-2) working in series configuration. System fails completely when either of the units gets failed. The repair time of unit-2 is considered to be much more as compared to the repair time of unit-1. So, to minimize the breakdown period of the system, a standby unit is provided against the second unit. Regenerative point technique (RPT) is used to develop a semi-markovian reliability model for the mentioned system. Optimum cut-off points concerning the profitability of the system have also been obtained. The model has applications in industries, particularly in aluminum industry.
\end{abstract}

Keywords: Dissimilar units, Optimum cut-off points, Repair time dependent standby, semi-Markov process, Series configuration

\section{INTRODUCTION}

The industries/organizations are now being modernized and focused on producing more reliable systems with increased availability and lesser break down time to achieve the set target. Redundancy is one of the most effective techniques, which may be used to enhance the performability of industrial systems and such systems have been analyzed by various researchers. Mokaddis et al. [1] analyzed standby system with three different operative stages. Parashar and Taneja [2] dealt with PLC hot standby system. A standby system with general life and repair time distribution was studied by Bieth et al. [3].Mahmoud and Mosherf [4] discussed different types of failure and preventive maintenance in their study. Malhotra and Taneja [5] stochastically analysed a system wherein operability of more than one unit depends upon requirement. Manocha and Taneja [6] took arbitrary distribution for all random variables. El- Sherbeny [7] studied such systems with the concept of random change of units. Manocha et al. [8] investigated database system keeping hot standby unit under constant observation.

Dissimilar units system may also be observed in the industrial sector. Mokkadis et al. [9] analysed a system by considering two types of repair and inspection of failed unit.

Revised Manuscript Received on February 18, 2020.

* Correspondence Author

Jai Bhagwan, Department of Mathematics, Government P.G. Nehru College Jhajjar, Haryana, India. Email: jaichaudhary81@gmail.com

Amit Manocha*, Department of Mathematics, TIT\&S, Bhiwani, Haryana, India. Email: amitmanocha80@yahoo.com

Anil Taneja, Department of Mathematics, Skyline University Nigeria, Kano,Nigeria, Email:dr.anilkrtaneja@gmail.com

(c) The Authors. Published by Blue Eyes Intelligence Engineering and Sciences Publication (BEIESP). This is an open access article under the CC BY-NC-ND license (http://creativecommons.org/licenses/by-nc-nd/4.0/)
Sadeghi and Roghanian [10] studied two unit warm standby system by considering two dissimilar units with imperfect switching mechanism. Rahbi et al. [11] did the reliability analysis of rodding anode plant consisting of eight dissimilar units used in aluminum industry. Chopra and Ram[12] carried out reliability analysis of two dissimilar units parallel system using Gumbel-Hougaard family copula. In a two dissimilar unit series system, it may be observed that one of the two dissimilar units, whenever gets failed, may require more time to get repaired as compared to the other. These types of systems are used at a large scale in network communication, textile industry, aluminum industry etc. For such systems, if a unit gets failed, the whole system becomes non-functional and hence introduction of a standby unit may reduce the frequency of breakdowns. However, using standby units against both the units may be a costly affair. Therefore, to keep a balance between the cost of using standby units and breakdown time of the systems, one may use single standby unit against that unit whose recovery time after failure is more than the other.

The present study is an attempt to stochastically analyse a system comprising two dissimilar units connected in series (unit-1/unit-2), where a standby unit is kept against the second unit. In the system under consideration, let us assume that unit- 2 takes more time to repair on failure as compared to the repair time of the first unit and hence breakdown period of system is much more in case of the failure of second unit. To reduce breakdown period of the system a standby unit is installed against unit-2.The product being manufactured by such a system is assumed to be first processed on unit- 1 and then on unit-2. System fails completely if either unit-1 or unit-2 along with its standby after putting it into operation gets failed. The technique and the other assumptions taken in the present study are same as that taken in [2].Optimum cut-off points for various costs which affect the profitability of the system have also been obtained.

\section{Notations}

$\mathrm{O}_{1} / \mathrm{O}_{2} \quad$ operative unit-1 / unit-2

$\mathrm{S}_{2} \quad$ standby unit for unit-2

$\omega_{1} / \omega_{2} \quad$ constant failure rate of unit-1and 2

$\mathrm{F}_{\mathrm{r} 1} / \mathrm{F}_{\mathrm{Wr} 1} \quad$ unit-1 under repair/ waiting for repair

$\mathrm{F}_{\mathrm{r} 2} / \mathrm{F}_{\mathrm{R} 2} / \mathrm{F}_{\mathrm{Wr} 2}$ unit-2 under repair/repair from previous state/ waiting for repair

$\mathrm{D}_{1} / \mathrm{D}_{2} \quad$ down unit-1/unit-2

$\mathrm{g}_{1}(\mathrm{t}) / \mathrm{g}_{2}(\mathrm{t})$ density function of repair time for unit-1and 2 Note: For some other notations one may refer to [2] and [5].

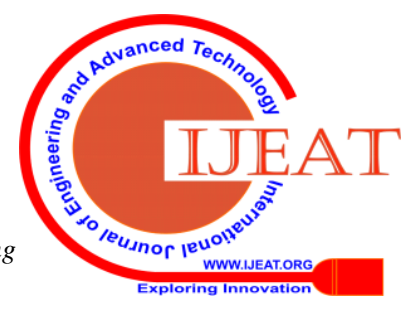




\section{TRANSITION DENSITIES \& MEAN SOJOURN TIMES}

Possible transitions for the model are shown in Fig.1.All the states except 3 and 4 are regenerative states.

States 0 and 2 are up, whereas 1, 3 and 4 are failed states.

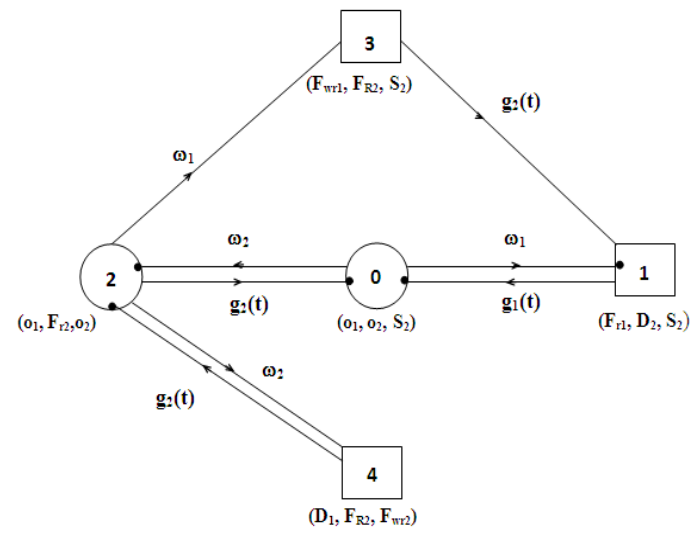

Operative state

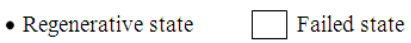

Fig.1. Transition diagram

Transition densities are:

$\mathrm{q}_{01}(\mathrm{t})=\omega_{1} \mathrm{e}^{-\left(\omega_{1}+\omega_{2}\right) \mathrm{t}}, \quad \mathrm{q}_{02}(\mathrm{t})=\omega_{2} \mathrm{e}^{-\left(\omega_{1}+\omega_{2}\right) \mathrm{t}}$,

$\mathrm{q}_{10}(\mathrm{t})=\mathrm{g}_{1}(\mathrm{t}) \quad, \quad \mathrm{q}_{20}(\mathrm{t})=\mathrm{e}^{-\left(\omega_{1}+\omega_{2}\right) \mathrm{t}} \mathrm{g}_{2}(\mathrm{t})$,

$q_{21}^{(3)}(t)=\left(\omega_{1} e^{-\left(\omega_{1}+\omega_{2}\right) t}(C)\right) g_{2}(t)$,

$\mathrm{q}_{22}^{(4)}(\mathrm{t})=\left(\omega_{2} \mathrm{e}^{-\left(\omega_{1}+\omega_{2}\right) \mathrm{t}}(\mathrm{C}) 1\right) \mathrm{g}_{2}(\mathrm{t})$,

$\mathrm{q}_{23}(\mathrm{t})=\omega_{1} \mathrm{e}^{-\left(\omega_{1}+\omega_{2}\right) t} \overline{\mathrm{G}}_{2}(\mathrm{t})$

By the probabilistic argument, the non-zero elements $\mathrm{p}_{\mathrm{ij}}$ are

obtained as: $\quad \mathrm{p}_{\mathrm{ij}}=\lim _{\mathrm{s} \rightarrow 0} \mathrm{q}_{\mathrm{ij}}^{*}(\mathrm{~s})$

For the developed model, mean sojourn time and contribution to sojourn time are mathematically expressed as:

$\mu_{0}=\int_{0}^{\infty} \mathrm{e}^{-\left(\omega_{1}+\omega_{2}\right) t} \mathrm{dt}, \quad \mu_{2}=\int_{0}^{\infty} \mathrm{e}^{-\left(\omega_{1}+\omega_{2}\right) t} \overline{G_{2}(t)} d t$

Thus, $\quad \mathrm{m}_{01}+\mathrm{m}_{02}=\mu_{0}, \quad \mathrm{~m}_{10}=-\mathrm{g}_{1}^{*}(0)=\mathrm{K}_{1}$ (say), $\mathrm{m}_{20}+\mathrm{m}_{23}+\mathrm{m}_{24}=\mu_{2}, \quad \mathrm{~m}_{20}+\mathrm{m}_{21}^{(3)}+\mathrm{m}_{22}^{(4)}=-\mathrm{g}_{2}^{* \prime}(0)=\mathrm{K}_{2}$.

\section{RELIABILITY AND MTSF}

If $\mathrm{rt}_{0}(\mathrm{t})$ and $\mathrm{rt}_{2}(\mathrm{t})$ denotes the CDF of first passage time from

$\mathrm{rt}_{0}(\mathrm{t})=\mathrm{Q}_{01}(\mathrm{t})+\mathrm{Q}_{02}(\mathrm{t}){ }^{\circledR} \mathrm{rt}_{2}(\mathrm{t})$

$\mathrm{rt}_{2}(\mathrm{t})=\mathrm{Q}_{23}(\mathrm{t})+\mathrm{Q}_{24}(\mathrm{t})+\mathrm{Q}_{20}(\mathrm{t}){ }^{\circledR} \mathrm{rt}_{0}(\mathrm{t})$

Thus, the reliability of the system

$\mathrm{R}(\mathrm{t})=\mathrm{L}^{-1}[\{\mathrm{D}(\mathrm{s})-\mathrm{N}(\mathrm{s})\} / \mathrm{sD}(\mathrm{s})]$

and

$\operatorname{MTSF}=\int_{0}^{\infty} R(t) d t=N / D$ states 0 and 2 to a failed state respectively, then we have

where

$$
\begin{aligned}
& \mathrm{N}(\mathrm{s})=\left\{\omega_{1} /\left(\mathrm{s}+\omega_{1}+\omega_{2}\right)\right\} \\
& +\left\{\omega_{2}\left(\omega_{1}+\omega_{2}\right) /\left(\mathrm{s}+\omega_{1}+\omega_{2}\right)^{2}\right\}\left\{1-\mathrm{g}_{2}^{*}\left(\mathrm{~s}+\omega_{1}+\omega_{2}\right)\right\} \\
& \mathrm{D}(\mathrm{s})=1-\left\{\omega_{2} \mathrm{~g}_{2}^{*}\left(\mathrm{~s}+\omega_{1}+\omega_{2}\right) /\left(\mathrm{s}+\omega_{1}+\omega_{2}\right)\right\} \\
& \mathrm{N}=\mu_{0}+\mathrm{p}_{02} \mu_{2} \text {, } \\
& \mathrm{D}=1-\mathrm{P}_{02} \mathrm{P}_{20}
\end{aligned}
$$

\section{AVAILABILITY ANALYSIS}

The recursive relations for point-wise availability $\mathrm{up}_{\mathrm{i}}(\mathrm{t})$, $\mathrm{i}=0,1,2$ are:

$\mathrm{up}_{0}(\mathrm{t})=\mathrm{a}_{0}(\mathrm{t})+\mathrm{q}_{01}(\mathrm{t}) \subset \mathrm{up}_{1}(\mathrm{t})+\mathrm{q}_{02}(\mathrm{t}) \subseteq \mathrm{up}_{2}(\mathrm{t})$

$\mathrm{up}_{1}(\mathrm{t})=\mathrm{q}_{10}(\mathrm{t}) \subset \mathrm{up}_{0}(\mathrm{t})$

$\mathrm{up}_{2}(\mathrm{t})=\mathrm{a}_{2}(\mathrm{t})+\mathrm{q}_{20}(\mathrm{t})\left(\mathrm{up}_{0}(\mathrm{t})+\mathrm{q}_{21}^{(3)}\left(\mathrm{C} \mathrm{up}_{1}(\mathrm{t})\right.\right.$

$+\mathrm{q}_{22}^{(4)}\left(\mathrm{up}_{2}(\mathrm{t})\right.$

where $\mathrm{a}_{0}(\mathrm{t})=\mathrm{e}^{-\left(\omega_{1}+\omega_{2}\right) \mathrm{t}}, \quad \mathrm{a}_{2}(\mathrm{t})=\mathrm{e}^{-\left(\omega_{1}+\omega_{2}\right) t} \overline{\mathrm{G}_{2}(\mathrm{t})}$

Thus, as time $\mathrm{t}$ approaches to infinity the availability is $\mathrm{up}_{0}=\limsup _{\mathrm{s} \rightarrow 0}^{* *}(\mathrm{~s})=\operatorname{lims}_{\mathrm{s} \rightarrow 0} \mathrm{~s}_{1}(\mathrm{~s}) / \mathrm{D}_{1}(\mathrm{~s})=\mathrm{N}_{1} / \mathrm{D}_{1}$

where $\mathrm{N}_{1}(\mathrm{~s})=\left\{1-\mathrm{q}_{22}^{(4) *}(\mathrm{~s})\right\} \mathrm{a}_{0}{ }^{*}(\mathrm{~s})+\mathrm{q}_{02}{ }^{*}(\mathrm{~s}) \mathrm{a}_{2}{ }^{*}(\mathrm{~s})$,

$$
\begin{aligned}
& \mathrm{D}_{1}(\mathrm{~s})=\left\{1-\mathrm{q}_{01}{ }^{*}(\mathrm{~s}) \mathrm{q}_{10}{ }^{*}(\mathrm{~s})\right\}\left\{1-\mathrm{q}_{22}^{(4) *}(\mathrm{~s})\right\} \\
& \quad-\mathrm{q}_{02}{ }^{*}(\mathrm{~s})\left\{\mathrm{q}_{10}{ }^{*}(\mathrm{~s}) \mathrm{q}_{21}^{(3) *}(\mathrm{~s})+\mathrm{q}_{20}{ }^{*}(\mathrm{~s})\right\}, \\
& \mathrm{N}_{1}=\left(1-\mathrm{p}_{22}^{(4)}\right) \mu_{0}+\mathrm{p}_{02} \mu_{2}, \\
& \mathrm{D}_{1}=\left(1-\mathrm{p}_{22}^{(4)}\right)\left(\mu_{0}+\mathrm{p}_{01} \mathrm{~K}_{1}\right)+\mathrm{p}_{02} \mathrm{~K}_{2} .
\end{aligned}
$$

\section{BUSY PERIOD ANALYSIS}

The system of equations obtained for evaluating busy period of repairman $b_{i}(t), i=0,1,2$ are:

$\mathrm{bt}_{0}(\mathrm{t})=\mathrm{q}_{01}(\mathrm{t})\left(\mathrm{bt}_{1}(\mathrm{t})+\mathrm{q}_{02}(\mathrm{t})\left(\mathrm{bt}_{2}(\mathrm{t})\right.\right.$

$\mathrm{bt}_{1}(\mathrm{t})=\mathrm{l}_{1}(\mathrm{t})+\mathrm{q}_{10}(\mathrm{t})\left(\mathrm{C} \mathrm{bt}_{0}(\mathrm{t})\right.$

$\mathrm{bt}_{2}(\mathrm{t})=\mathrm{l}_{2}(\mathrm{t})+\mathrm{q}_{20}(\mathrm{t})\left(\mathrm{bt}_{0}(\mathrm{t})+\mathrm{q}_{21}^{(3)}\left(\mathrm{C} \mathrm{bt}_{1}(\mathrm{t})\right.\right.$

$$
+\mathrm{q}_{22}^{(4)}\left(\mathrm{C} \mathrm{bt}_{2}(\mathrm{t})\right.
$$

where $\mathrm{l}_{1}(\mathrm{t})=\overline{\mathrm{G}_{1}}(\mathrm{t}), \quad \mathrm{l}_{2}(\mathrm{t})=\overline{\mathrm{G}}_{2}(\mathrm{t})$

Thus, in steady-state, we have

$\mathrm{bt}_{0}=\lim _{\mathrm{s} \rightarrow 0}\left\{\mathrm{~s} \mathrm{~N}_{2}(\mathrm{~s}) / \mathrm{D}_{1}(\mathrm{~s})\right\}=\mathrm{N}_{2} / \mathrm{D}_{1}$

where

$$
\begin{aligned}
\mathrm{N}_{2}(\mathrm{~s}) & =\left\{\mathrm{q}_{01}{ }^{*}(\mathrm{~s})-\mathrm{q}_{01}{ }^{*}(\mathrm{~s}) \mathrm{q}_{22}^{(4) *}(\mathrm{~s})+\mathrm{q}_{02}{ }^{*}(\mathrm{~s}) \mathrm{q}_{21}^{(3) *}(\mathrm{~s})\right\} \mathrm{l}_{1}{ }^{*}(\mathrm{~s}) \\
& +\mathrm{q}_{02}{ }^{*}(\mathrm{~s}) \mathrm{l}_{2}{ }^{*}(\mathrm{~s}) \\
\mathrm{N}_{2}= & \left\{\left(1-\mathrm{p}_{22}^{(4)}\right) \mathrm{p}_{01}+\mathrm{p}_{02} \mathrm{p}_{21}^{(3)}\right\} \mathrm{K}_{1}+\mathrm{p}_{02} \mathrm{~K}_{2}
\end{aligned}
$$

\section{EXPECTED NUMBER of VISITS BY REPAIRMAN}

The equations for obtaining expected number of visits by repairman $\mathrm{ev}_{\mathrm{i}}(\mathrm{t}), \mathrm{i}=0,1,2$ in specific unit of time, are: $\mathrm{ev}_{0}(\mathrm{t})=\mathrm{Q}_{01}(\mathrm{t}) \stackrel{\circledR}{\circledR}\left\{\mathrm{ev}_{1}(\mathrm{t})+1\right\}+\mathrm{Q}_{02}(\mathrm{t}) \stackrel{B}{\circledR}\left\{\mathrm{ev}_{2}(\mathrm{t})+1\right\}$ $\mathrm{ev}_{1}(\mathrm{t})=\mathrm{Q}_{10}(\mathrm{t}){ }^{\circledR} \mathrm{ev}_{0}(\mathrm{t})$ $\mathrm{ev}_{2}(\mathrm{t})=\mathrm{Q}_{20}(\mathrm{t}){ }^{\circledR} \mathrm{e} \mathrm{v}_{0}(\mathrm{t})+\mathrm{Q}_{21}^{(3)}(\mathrm{t}){ }^{\circledR} \mathrm{ev}_{1}(\mathrm{t})+\mathrm{Q}_{22}^{(4)}(\mathrm{t}){ }^{\circledR} \mathrm{ev}_{2}(\mathrm{t})$

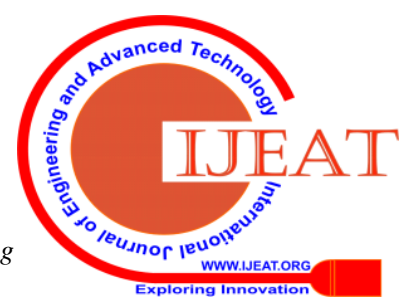


In long run

$\mathrm{ev}_{0}=\lim _{\mathrm{s} \rightarrow 0}\left\{\mathrm{~s} \mathrm{~N}_{3}(\mathrm{~s}) / \mathrm{D}_{1}(\mathrm{~s})\right\}=\mathrm{N}_{3} / \mathrm{D}_{1}$

where $\mathrm{N}_{3}(\mathrm{~s})=\left\{1-\mathrm{Q}_{22}^{(4) * *}(\mathrm{~s})\right\}\left\{\mathrm{Q}_{01}{ }^{* *}(\mathrm{~s})+\mathrm{Q}_{02}{ }^{* *}(\mathrm{~s})\right\}$

$\mathrm{N}_{3}=\left(1-\mathrm{p}_{22}^{(4)}\right)$

\section{PROFIT ANALYSIS}

The profit equation, therefore, is

$$
\mathrm{P}_{0}=\mathrm{C}_{\mathrm{R}} \mathrm{P}_{0}-\mathrm{C}_{\mathrm{B}} \mathrm{bt}_{0}-\mathrm{C}_{\mathrm{V}} \mathrm{ev}_{0}
$$

where $C_{R}=$ Revenue per unit up time

$\mathrm{C}_{\mathrm{B}}=$ Cost per unit time for engaging repair facility

$\mathrm{C}_{\mathrm{V}}=$ Repairman charges for each visit

\section{RESULT AND DISCUSSION}

Letting $g_{1}(t)=\alpha_{1} e^{-\alpha_{1} t}, g_{2}(t)=\alpha_{2} e^{-\alpha_{2} t}$; considering $\omega_{2}=$ 0.005 per hr, $\alpha_{1}=0.2$ per hr; and varying $\omega_{1}, \alpha_{2}$; the values of MTSF and availability are tabulated as:

Table-I: Values of MTSF and Availability (upo) w.r.t. $\omega_{1}$ and $\alpha_{2}$

\begin{tabular}{|c|c|c|c|c|c|c|}
\hline \multirow{2}{*}{$\omega_{1}$} & \multicolumn{2}{|c|}{$\alpha_{2}=\mathbf{0 . 1}$ (per hr) } & \multicolumn{2}{c|}{$\alpha_{2}=\mathbf{0 . 1 5}$ (per hr) } & \multicolumn{3}{c|}{$\alpha_{2}=\mathbf{0 . 2}$ (per hr) } \\
\cline { 2 - 7 } (per hr) & $\begin{array}{c}\text { MTSF } \\
\text { (In hrs) }\end{array}$ & $\begin{array}{c}\text { Availability } \\
\text { (up } \text { ) }\end{array}$ & $\begin{array}{c}\text { MTSF } \\
\text { (In hrs) }\end{array}$ & $\begin{array}{c}\text { Availability } \\
\text { (up })\end{array}$ & $\begin{array}{c}\text { MTSF } \\
\text { (In hrs) }\end{array}$ & $\begin{array}{c}\text { Availability } \\
\text { (up })^{\text {) }}\end{array}$ \\
\hline 0.001 & 828.77 & 0.9925 & 865.6 & 0.9939 & 894.07 & 0.9944 \\
\hline 0.002 & 453.53 & 0.9874 & 464.18 & 0.9890 & 472.16 & 0.9895 \\
\hline 0.003 & 312.18 & 0.9823 & 317.12 & 0.9840 & 320.78 & 0.9846 \\
\hline 0.004 & 238 & 0.9773 & 240.82 & 0.9792 & 242.91 & 0.9798 \\
\hline 0.005 & 192.31 & 0.9724 & 194.12 & 0.9744 & 195.45 & 0.9750 \\
\hline 0.006 & 161.33 & 0.9675 & 162.59 & 0.9696 & 163.51 & 0.9703 \\
\hline
\end{tabular}

Graphs of profit $\left(\mathrm{P}_{0}\right)$ with respect to the following have been plotted in Figs. 2 and 3.

i) $C_{R}$ for varied $\omega_{1}$

ii) $C_{B}$ for varied $C_{V}$

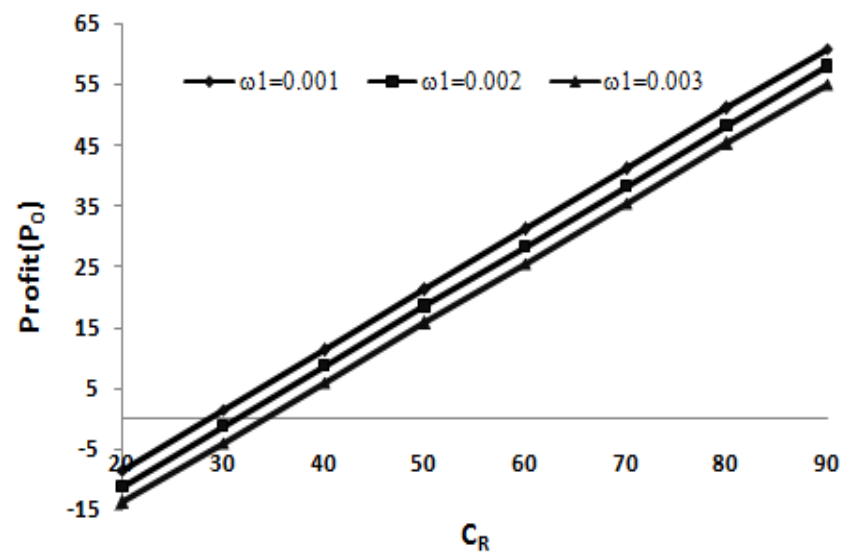

Fig.2. Profit $\left(\mathbf{P}_{0}\right)$ versus $\left(C_{R}\right)$

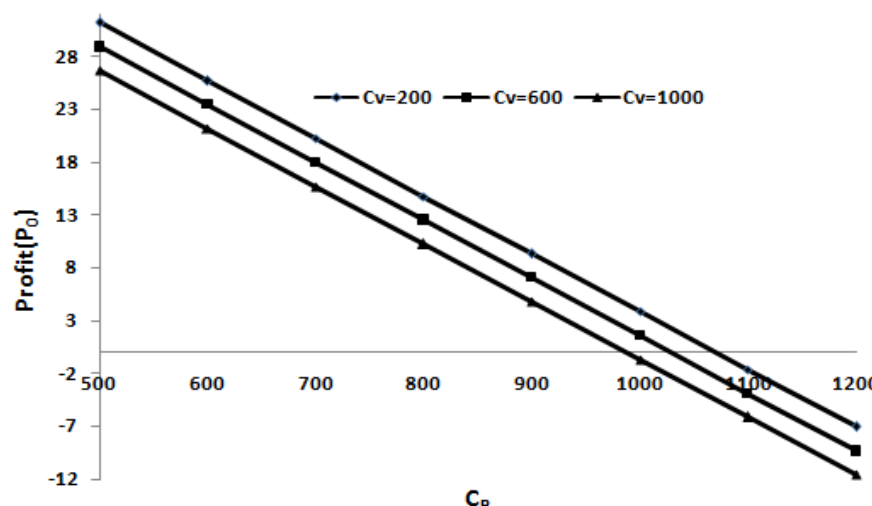

Fig.3. Profit $\left(\mathbf{P}_{0}\right)$ versus cost $\left(\mathrm{C}_{B}\right)$

The above figures reveal what has been tabulated as follows:
Table-II: Conditions for the profitability of the system

\begin{tabular}{|l|l|l|l|}
\hline $\begin{array}{l}\text { Assumed } \\
\text { parametric } \\
\text { values }\end{array}$ & $\begin{array}{l}\text { Varied } \\
\text { parameter }\end{array}$ & $\begin{array}{l}\text { Condition for the system } \\
\text { to be } \\
\text { profitable(Optimum } \\
\text { cut-off points) }\end{array}$ & Remark \\
\hline $\begin{array}{l}\omega_{2}=0.005, \\
\alpha_{1}=0.2,\end{array}$ & $\omega_{1}=0.001$ & $\mathrm{C}_{\mathrm{R}}>28.64$ & $\begin{array}{l}\text { Otherwise, } \\
\text { system will } \\
\alpha_{2}=0.1,\end{array}$ \\
\cline { 2 - 3 } $\begin{array}{l}\mathrm{C}_{\mathrm{B}}=500, \\
\mathrm{C}_{\mathrm{V}}=200\end{array}$ & $\omega_{1}=0.002$ & $\mathrm{C}_{\mathrm{R}}>31.33$ & put to a loss \\
\cline { 2 - 3 }$\omega_{1}=0.001$, & $\omega_{1}=0.003$ & $\mathrm{C}_{\mathrm{R}}>34.03$ & $\begin{array}{l}\text { Otherwise, } \\
\text { system will } \\
\omega_{2}=0.005,\end{array}$ \\
\cline { 2 - 3 }$\alpha_{1}=0.2$, & $\mathrm{C}_{\mathrm{V}}=600$ & $\mathrm{C}_{\mathrm{B}}<1070.12$ & \\
$\alpha_{2}=0.1$, & $\mathrm{C}_{\mathrm{B}}<1028.54$ & \\
$\mathrm{C}_{\mathrm{R}}=60$ & $\mathrm{C}_{\mathrm{V}}=1000$ & $\mathrm{C}_{\mathrm{B}}<986.96$ & \\
\hline
\end{tabular}

\section{CONCLUSION}

The stochastic analysis is carried out for a system comprising two dissimilar units connected in series with a standby unit against that unit which has more recovery time after failure. Cost is always a crucial factor for any industry/organization and hence cut-off points for revenue/cost have been determined, finding numerical results for a particular case, which may be used to assess as to what value of the parameter under consideration should be taken in order to have a profitable system. Cost analysis may be done for other parameters of interest also in the similar way by the users of such systems.

\section{REFERENCES}

G.S.Mokaddis, S.W. Labib, and A.M. Ahmed, "Analysis of a two-unit warm standby system subject to degradation," Microelectron. Reliab., vol.37 (4), 1997, pp.641-648.

2. B.Parashar, and G. Taneja, "Reliability and profit evaluation of a plc hot standby system based on a master- slave concept and two types of repair facilities," IEEE T. Reliab., vol.56 (3), 2007, pp. 534-539.

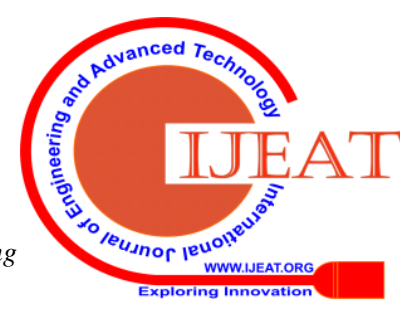


3. B. Beith, L. Hong, and J. Sarkar, "A standby system with two repair persons under arbitrary life-and repair times," Math. Comput. Modelling, vol. 51, 2010, pp. 756-767.

4. M.A.W. Mahmoud, and M.E. Mosherf, "On a two- unit cold standby system considering hardware, human error failures and preventive maintenance," Math. Comput. Modelling, vol. 51, 2010, pp. 736-745.

5. R. Malhotra, and G. Taneja, "Stochastic analysis of a two-unit cold standby system wherein both units may become operative depending upon the demand," JQRE, 2014, pp.1-13.

6. A. Manocha, and G.Taneja, "Stochastic analysis of a two-unit cold standby system with arbitrary distribution for life, repair and waiting times," IJPE, vol. 11(3), 2015, pp. 293-299.

7. M.S. El-Sherbeny, "Stochastic analysis of a system with cold standby, general distribution and random change in units," AMIS, vol.10 (2), 2016, pp. 565-570.

8. A. Manocha, S.Singh, and A. Taneja, "Analysis of hot standby database system with standby unit under constant observation,"AJMI, vol.10 (1), 2018, pp. 25-32.

9. G.S. Mokaddis , G.S.Khalil, and H. Alhajri, Analysis of two dissimilar - unit cold standby redundant system subject to inspection and two types of repair," IJMER, vol.6(5), 2016, pp. 38-54.

10. M.Sadeghi, and E. Roghnian, "Reliability analysis of a warm standby repairable system with two cases of imperfect switching mechanism,' Scientia Iranica E, vol. 24(2), 2017, pp.808-822.

11. Y. A. Rahbi, S. M. Rizwan, B. M. Alkali, A. Cowell and G. Taneja, "Reliability analysis of rodding anode plant in aluminum industry," IJAER,vol 12(16), 2017, pp. 5616-5623.

12. G. Chopra, and M. Ram, "Reliability measures of two dissimilar units parallel system using gumbel-hougaard family copula", IJMEMS, vol. 4(1), 2019, pp. 116-130.

\section{AUTHORS PROFILE}

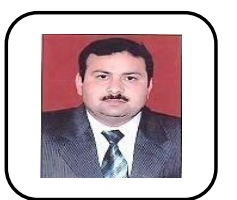

Jai Bhagwan is an Assistant Professor in Mathematics at Government P.G. Nehru College, Jhajjar, Haryana. He received his $\mathrm{PhD}$ in Mathematics from B.R.A. University, Agra. During 16 years of his teaching and research experience, he published around 9 research papers in journals of repute and contributed in 27 national/international conferences/seminar/workshop. He is in editorial committee of "Annals of Mathematical Physics": an International Journal by Serial Publications. He coauthored three books on Applied Mathematics and his area of interest is reliability modelling.

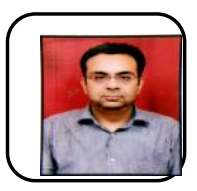

Amit Manocha is serving as an Assistant Professor(Mathematics) in Department of Applied Sciences at TIT\&S, Bhiwani, Haryana, India. He has been teaching undergraduate/postgraduate engineering students since 17 years. He pursued his doctorate in Mathematics from NIT, Kurukshetra. Over 14 research papers in various reputed journals/proceedings owe to his credit. He has actively contributed and participated in various academic events like conferences, seminars etc His research areas are reliability modeling and applied mathematics.

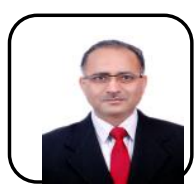

Anil Taneja is a Professor and Head in the Department of Mathematics at Skyline University Nigeria, Kano (Nigeria). He qualified UGC-NET examination (mathematics) in year 2000. He did his PhD in the area of Reliability modelling from M.D. University, Rohtak, India. He published 12 research papers in national/international journals of repute and contributed in 20 national/international conferences/seminars. He has delivered expert lectures and chaired sessions in various conferences/seminars/workshops. With 25 years of teaching and administrative experience, he is actively involved in research areas like reliability modeling, queuing theory and information theory. 